\title{
Editorial
}

\section{New Advances in Diagnosis and Management of Glaucoma}

\author{
M. Reza Razeghinejad ${ }^{*}{ }^{\S}$ and Mohammad H. Nowroozzadeh
}

Poostchi Eye Research Center, Shiraz University of Medical Sciences, Shiraz, Iran

Glaucoma is the first cause of unemendable visual disability and blindness worldwide [1]. If glaucoma is detected at early stages, the visual disability can be prevented or postponed, and consequently its social, economic, and psychic burdens diminished. Although glaucoma is a complex and poorly understood disorder, the primary goal of therapy is lowering Intraocular Pressure (IOP), which is not only one of the most significant risk factors in development of the disease, but also the only modifiable one.

Glaucoma is a chronic disease that must be longitudinally assessed and treated based on the appearance of the optic nerve head and evaluation of its function. Traditionally, optic disc evaluation and visual field test along with IOP measurement have been used for glaucoma diagnosis and monitoring. These tests are still of great value in the field of glaucoma, but are subjected to considerable measurement errors, because visual field is a subjective test that requires patient cooperation, and optic disc evaluation is an objective but observer-dependent test. Although visual field testing has been widely used for diagnosis, staging and monitoring the disease, in many patients visual field losses only become detectable after a substantial number of optic nerve fibers has been lost. Therefore, early identification of structural damage to the optic disc and retinal nerve fiber layer is paramount for an early diagnosis of the disease. Heidelberg Retina Tomograph is an objective, observer-independent method for measurement of optic disc parameters, which allows detecting subtle changes in optic nerve head configuration, and hence earlier detection of glaucoma progression [2]. Optical coherence tomography can reliably measure peripapillary retinal nerve fiber layer and macular thickness as an anatomical proxy for visual field test. This test is objective, highly repeatable, and requires less patient cooperation than visual field. Other limitations of visual field such as low vision are also less problematic in these objective tests $[3,4]$. However, these devices have their own limitations which should be taken into account while interpreting them.

*Address correspondence to this author at the Poostchi Eye Research Center, Shiraz University of Medical Sciences, Shiraz, Iran;

Tel/Fax: +98-71-32302830; E-mail: razeghinejad@yahoo.com,

${ }^{\S}$ Guest Editor
With few exceptions, medical therapy is the first treatment modality in glaucoma patients. Despite a number of pharmacological options, failure of medical treatment is a significant issue owing to drug ineffectiveness and intolerance, but also to poor patient compliance and persistence. Although after introduction of new drugs in mid-1990s, the global number of glaucoma operations has decreased, particularly in the western countries [1], we still need medications with higher potency and fewer side effects. Considering glaucoma as a neurodegenerative disorder, new efforts have been made to prevent or reverse retinal nerve fiber layer loss through neuroprotective or neuroregenerative therapies [5].

Patients with an uncontrolled IOP despite implementing medical and laser therapy need a surgical intervention. For several decades, trabeculectomy and tube shunt have been regarded as milestones of glaucoma surgery. In spite of modifications applied to these procedures to enhance their efficacy and minimize their complications, none of them is perfect and the new glaucoma surgeries do not seem to be a perfect substitution [6]. The pediatric groups of glaucoma patients have their own characteristics and need proper approach in terms of medical and surgical treatment [7].

As the technology advances, and our understanding of glaucoma improves, novel tools and methods are being available for a better diagnosis, monitoring, and treatment of glaucoma. The constellation of review articles that have been gathered in this issue are intended to provide updated information on new advances in different aspects of glaucoma diagnosis and treatment.

\section{REFERENCES}

[1] Rachmiel R, Trope GE, Chipman ML, et al. Effect of medical therapy on glaucoma filtration surgery rates in Ontario. Arch Ophthalmol 2006; 124(10): 1472-7.

[2] Maslin JS, Mansouri K, Dorairaj SK. HRT for the diagnosis and detection of glaucoma progression. Open Ophthalmol J 2015; 9: 58-67.

[3] Gracitelli CPB, Abe RY, Medeiros FA. Spectral-domain optical coherence tomography for glaucoma diagnosis. Open Ophthalmol J 2015; 9: 68-77.

[4] Abe RY, Gracitelli CPB, Medeiros FA The use of spectral-domain optical coherence tomography to detect glaucoma. Open Ophthalmol J 2015; 9: 78-88.

[5] Kolko M. Present and new treatment strategies in the management of glaucoma. Open Ophthalmol J 2015; 9: 89-100. 
[6] Spaeth GL, Cvintal V, Figueiredo A. Is there a need for new surgical procedures for glaucoma? yes! Open Ophthalmol J 2015; 9: 101-3.
[7] Khan AO. A Surgical approach to pediatric glaucoma. Open Ophthalmol J 2015; 9: 104-12

(C) Razeghinejad and Nowroozzadeh; Licensee Bentham Open.

This is an open access article licensed under the terms of the Creative Commons Attribution Non-Commercial License (http://creativecommons.org/licenses/by-nc/3.0/) which permits unrestricted, non-commercial use, distribution and reproduction in any medium, provided the work is properly cited. 